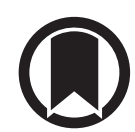

CrossMark

\title{
The European Network for ICU-Related Respiratory Infections (ENIRRIs) ERS Clinical Research Collaboration
}

\author{
Ignacio Martin-Loeches ${ }^{1,2}$ and Antoni Torres ${ }^{3,4}$ on behalf of the ENIRRIs \\ Clinical Research Collaboration
}

Affiliations: 'St James's Hospital, Multidisciplinary Intensive Care Research Organization (MICRO), Dublin, Ireland. ${ }^{2}$ Centros de Investigación Biomédica en Red (CIBER) de Enfermedades Respiratorias (CIBERES), Barcelona, Spain. ${ }^{3}$ Pulmonary Intensive Care Unit, Respiratory Institute, Hospital Clinic of Barcelona, IDIBAPS, Barcelona, Spain. ${ }^{4}$ Universitat de Barcelona, Barcelona, Spain.

Correspondence: Ignacio Martin-Loeches, Multidisciplinary Intensive Care Research Organization (MICRO), St James's Hospital, P.0. Box 580, James's Street, Dublin 8, Ireland. E-mail: drmartinloechesdgmail.com

@ERSpublications

The European Network for ICU-Related Respiratory Infections (ENIRRIs) ERS Clinical Research Collaboration is addressing the priorities for respiratory infection research in critical care settings http://ow.ly/3KHa30mHryj

Cite this article as: Martin-Loeches I, Torres A. The European Network for ICU-Related Respiratory Infections (ENIRRIs) ERS Clinical Research Collaboration. Eur Respir J 2019; 53: 1801972 [https://doi.org/ 10.1183/13993003.01972-2018].

\section{Introduction}

Respiratory infections are by far the most challenging nosocomial complications in intensive care units (ICUs) [1]. The incidence rate of respiratory infections in ICUs has dramatically decreased over recent years, with an important component related to ventilator-associated pneumonia (VAP) episodes [2]. Regarding other respiratory infections occurring in ICUs, such as ventilator-associated tracheobronchitis (VAT), pneumonia in non-ventilated patients and pneumonia developed on the ward that requires ICU admission, the information is scarcer [3]. The complexity of infections in critical care is indicated diagrammatically in figure 1 . Several years ago, the European Respiratory Society (ERS) set out to provide scientific support for key areas of respiratory diseases, by establishing the ERS Clinical Research Collaborations (CRCs) programme to build and maintain European multicentre networks of researchers, from both within and outside of the Society [4].

\section{Rationale}

Regarding ICU pneumonia in non-ventilated patients, there is growing evidence that there are many similarities with VAP and treatment decisions should consider both [2]. The previous view of episodes being less severe compared to VAP has been shown not to be completely accurate [5]. It has been noted that the patients admitted to the ICU are older and present more comorbidities than previously, but despite patients being sicker, the mortality rate has decreased [6]. In addition, some patients that are admitted to the ICU do not have "full code" status (i.e. they have not agreed that all resuscitative and aggressive curative treatments can be provided), and they can be managed with different levels of care depending on the baseline and frailty status [7]. Therefore, the use of antibiotics is still the most important action when nosocomial infection happens, and invasive mechanical ventilation should be deferred or even avoided in many circumstances [8]. 


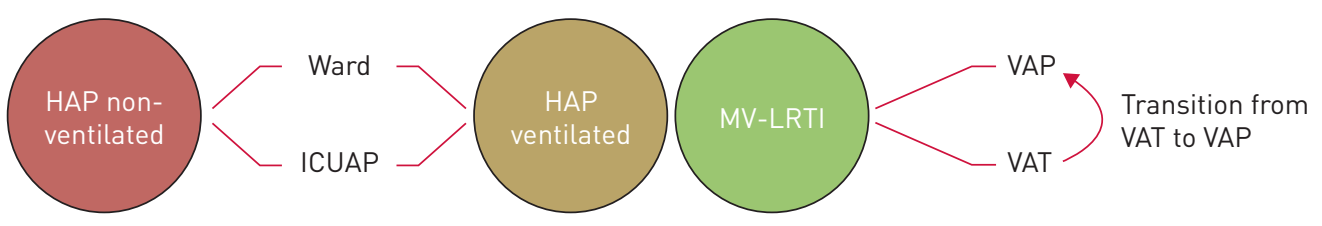

FIGURE 1 Complexity of infections in critical care. HAP: hospital-acquired pneumonia; ICUAP: intensive care unit-acquired pneumonia; MV-LRTI: mechanically ventilated lower respiratory tract infection; VAP: ventilator-associated pneumonia; VAT: ventilator-associated tracheobronchitis.

Nosocomial pneumonia occurring on the ward is frequent and is the second most frequent infection in hospital settings after urinary tract infections [9]. Interestingly, resource consumption and fatality associated with nosocomial pneumonia is far higher than for urinary tract infections. Because of the number of episodes and number of patients, which are much higher in the ward than in the ICU, there is a problem with prevention, diagnosis and treatment. It is also in the scope of some regulatory agencies to better understand the burden associated with these events. The mortality rates are likely to be different depending on whether 1) the patient develops nosocomial pneumonia on the ward and needs to be transferred over to the ICU for mechanical ventilation (ventilator-associated and hospital-acquired pneumonia (VHAP)), 2) the patient develops infections while in the ICU being ventilated (VAP) or not ventilated (hospital-acquired pneumonia, non-ventilated (HAPNV)), or 3) a patient develops VAT. Based on the difficulty in diagnosis, as some patients do not have the same number of diagnostic procedures, such as radiography, daily bloods, etc., the treatment is often delayed. The aetiology is an important point because, with the exception of some chronic respiratory and immunosuppressed patients, adequate respiratory samples are often not obtained [10]. It is also very relevant that many of the antibiotic choices that have been published in international guidelines, while they also refer to hospital-acquired pneumonia, are very heterogeneous and treatment choices are randomly assigned depending on the attending team [11]. The emergence of antibiotic stewardship teams has helped in making adequate antibiotic choices based on the patient's risk factors, shortening treatment duration and ensuring de-escalation in a timely manner [12].

\section{Aims and challenges}

The European Network for ICU-Related Respiratory Infections (ENIRRIs) CRC was created in 2015 with the participation of 10 European countries with a good geographic representation, under the ERS Respiratory Infections Assembly. The ENIRRIs CRC started with the aim of supporting experienced research groups in the fields of epidemiology, aetiology, microbiology, pathophysiology, clinical management and prognosis of respiratory infections affecting critically ill patients. In 2017, the ENIRRIs became a more global network, including countries in Latin America [13].

The aim of the network has been to tackle several questions regarding diagnosis, treatment and general management of critically ill patients affected with a respiratory tract infection. One of the lessons learned from the past when analysing results of international networks has been the role of differentiating risk factors that were not included in previous guidelines. A good example is the role of the ecology of the pathogens in decision-making, in order to allow a "think global, act local" approach [14]. This is particularly relevant in order to constrain antibiotic pressure in settings with reasonably low rates of resistant pathogens. In previous guidelines on treatment of nosocomial infections, there was a broad recommendation depending on risk factors such as early- versus late-onset episodes and previous antibiotic consumption. Nowadays, major guidelines, both American and European, have made recommendations based on organ severity, e.g. for acute kidney injury, acute respiratory distress syndrome and septic shock in the American guidelines, and for ecology and septic shock in the European guidelines, in order to determine the likelihood of potentially resistant pathogens $[11,15]$.

Particularly important in recent years has been the study of the continuum from colonisation to infection and from VAT to VAP. Recent research has shown that progression from VAT to VAP is frequent, based on prospective and retrospective studies. Appropriate antibiotic treatment has been repeatedly found as the only factor independently associated with reduced risk for transition from VAT to VAP [16-18]. The number of patients with VAT needed to treat to prevent one episode of VAP has been shown to be around five. VAT has been largely criticised as a non-existent entity and where there is no doubt that it exists, the criteria for diagnosis have not been widely agreed by all the scientific community. The lack of consolidation on radiography has been taken as the core element from diagnosis, along with other aspects such as inflammatory response, aetiology and sputum characteristics. There are also potential links between VAT diagnosis and higher antibiotic consumption, and terms have emerged that clearly don't 
help clinical decision-making, such as "ventilator-associated event" and "infection-related ventilatorassociated complication", which have been proposed just for epidemiological purposes. Another important point is that we now have a better physiopathological understanding of local infection and inflammation within the lower respiratory tract, and recently some studies have discovered gene expression routes that are linked to an inadequate host response $[19,20]$. This ultimately results in an impaired host response that can develop into either VAT or VAP and thus give rise to different associated morbidity and mortality rates.

In the last decade, resistant strains have been emerging, some of them resistant to all antibiotic classes [21]. Since the early 1990s, we have known of the emergence of a variety of $\beta$-lactamases with a wide spectra of substrate specificity, which illustrate the drug resistance mechanisms. Three major groups of these enzymes have usually been distinguished: class $\mathrm{C}$ cephalosporinases (AmpC), extended-spectrum $\beta$-lactamases and different types of $\beta$-lactamases with carbapenemase activity, of which the so-called class $B$ metallo- $\beta$-lactamases have been of the greatest concern in the last decade. In addition, the number of Gram-positive resistant pathogens has decreased, with some big concerns about the rising rates of both community and nosocomial methicillin-resistant Staphylococcus aureus a decade ago [22]. The ENIRRIs CRC will also attempt to determine the potential morbidity and mortality attributable to different grades of resistance, based on resistance grades for which there has so far been no consensus reached: terms such as "multidrug-resistant", "extreme-drug resistant", "extensive, extensively or extremely-drug resistant" and "pandrug-resistant", which characterise resistance in multidrug-resistant organisms. The main goal will also be to determine 28- and 90-day mortality depending on the resistance patterns and find out whether the microbiology is comparable in four different populations: HAPNV, VHAP, VAT and VAP.

\section{Past achievements and future plans}

In summary, the CRC has confirmed participation of centres of excellence from different countries to ensure wide representation and participation. The CRC has been working with a robust database that highlights the objectives of the ENIRRIs project. The website will facilitate secure data sharing through a secure web interface (called "Safe Haven"). The network has previously conducted a scoping review to develop common protocols for data collection, as well as agreements and tools for data sharing agenda under the ENIRRIs members' task force. Following network meetings, the network has included more than 800 patients so far, with respiratory infections in critical care settings. The CRC will write and submit a "roadmap", which will be a consensus document outlining the research priorities for respiratory infection research in critical care settings, such as the major concerns about multidrug-resistant pathogens. The network will work in collaboration with other organisations, industry and public funding agencies.

The first results of the ENIRRIs CRC, describing colonisation and infection and local epidemiology patterns, will be completed by the end of the study period and compiled in a final document. To join and get further information about ENIRRIs, please contact the authors of this editorial. There is a need to develop an ongoing and sustainable network to provide real-time data across critical care settings in high-, middle- and low-income countries, in accordance with current gross national income.

Conflict of interest: I. Martin-Loeches reports grants from MSD and personal fees from Polyphor and accelerate, outside the submitted work. A. Torres reports personal fees from MSD, Pfizer and Polyphor, outside the submitted work.

\section{References}

1 Martin-Loeches I, Povoa P, Rodríguez A, et al. Incidence and prognosis of ventilator-associated tracheobronchitis (TAVeM): a multicentre, prospective, observational study. Lancet Respir Med 2015; 3: 859-868.

2 Zilahi G, Artigas A, Martin-Loeches I. What's new in multidrug-resistant pathogens in the ICU? Ann Intensive Care 2016; 6: 96

3 Martin-Loeches I, Nseir S, Valles J, et al. From ventilator-associated tracheobronchitis to ventilator-associated pneumonia. Réanimation 2013; 22: 231-237.

4 Brightling C, Genton C, Bill W, et al. ERS Clinical Research Collaborations: underpinning research excellence. Eur Respir J 2018; 52: 1801534.

5 Righy C, do Brasil PEA, Vallés J, et al. Systemic antibiotics for preventing ventilator-associated pneumonia in comatose patients: a systematic review and meta-analysis. Ann Intensive Care 2017; 7: 67.

6 Martin-Loeches I, Wunderink RG, Nanchal R, et al. Determinants of time to death in hospital in critically ill patients around the world. Intensive Care Med 2016; 42: 1454-1460.

7 Capuzzo M, Garrouste-Orgeas M, Martin-Loeches I. What were you able to do in your daily life? Performance status for the critically ill patient. Intensive Care Med 2017; 43: 104-106.

8 Martin-Loeches I, Leone M, Madách K, et al. Antibiotic therapy in the critically ill - expert opinion of the Intensive Care Medicine Scientific Subcommittee of the European Society of Anaesthesiology. Eur J Anaesthesiol 2017; 34: 215-220.

9 Niederman MS, Martin-Loeches I, Torres A. The research agenda in VAP/HAP: next steps. Intensive Care Med 2017; 43: 1389-1391. 
10 Almansa R, Socias L, Sanchez-Garcia M, et al. Critical COPD respiratory illness is linked to increased transcriptomic activity of neutrophil proteases genes. BMC Res Notes 2012; 5: 401.

11 Torres A, Niederman MS, Chastre J, et al. International ERS/ESICM/ESCMID/ALAT guidelines for the management of hospital-acquired pneumonia and ventilator-associated pneumonia. Eur Respir J 2017; 50: 1700582.

12 Tiszai-Szucs T, Mac Sweeney C, Keaveny J, et al. Feasibility of antimicrobial stewardship (AMS) in critical care settings: a multidisciplinary approach strategy. Med Sci 2018; 6: E40.

13 De Pascale G, Ranzani OT, Nseir S, et al. Intensive care unit patients with lower respiratory tract nosocomial infections: the ENIRRIs project. ERJ Open Res 2017; 3: 00092-2017.

14 De Waele JJ, Bassetti M, Martin-Loeches I. Impact of de-escalation on ICU patients' prognosis. Intensive Care Med 2014; 40: 1583-1585.

15 Kalil AC, Metersky ML, Klompas M, et al. Management of adults with hospital-acquired and ventilator-associated pneumonia: 2016 Clinical Practice Guidelines by the Infectious Diseases Society of America and the American Thoracic Society. Clin Infect Dis 2016; 63: e61-e111.

16 Martin-Loeches I, Coakley JD, Nseir S. Should we treat ventilator-associated tracheobronchitis with antibiotics? Semin Respir Crit Care Med 2017; 38: 264-270.

17 Rodríguez A, Póvoa P, Nseir S, et al. Incidence and diagnosis of ventilator-associated tracheobronchitis in the intensive care unit: an international online survey. Crit Care 2014; 18: R32.

18 Nseir S, Martin-Loeches I, Makris D, et al. Impact of appropriate antimicrobial treatment on transition from ventilator-associated tracheobronchitis to ventilator-associated pneumonia. Crit Care 2014; 18: R129.

19 Almansa R, Wain J, Tamayo E, et al. Immunological monitoring to prevent and treat sepsis. Crit Care 2013; 17: 109.

20 Martin-Loeches I, Papiol E, Almansa R, et al. Intubated patients developing tracheobronchitis or pneumonia have distinctive complement system gene expression signatures in the pre-infection period: a pilot study. Med Intensiva 2012; 36: $257-263$.

21 Martin-Loeches I, Rodriguez AH, Torres A. New guidelines for hospital-acquired pneumonia/ventilator-associated pneumonia: USA vs Europe. Curr Opin Crit Care 2018; 24: 347-352.

22 Vallés J, Martin-Loeches I, Torres A, et al. Epidemiology, antibiotic therapy and clinical outcomes of healthcare-associated pneumonia in critically ill patients: a Spanish cohort study. Intensive Care Med 2014; 40: $572-581$. 